\title{
Monitoring Burst (M-Burst)- A Novel Framework of Failure Localization in All-Optical Mesh Networks
}

\author{
Mohammed L. Ali*, Pin-Han $\mathrm{Ho}^{\dagger}$, Bin $\mathrm{Wu}^{\dagger}$, Janos Tapolcai ${ }^{\ddagger}$, and Basem Shihada ${ }^{\S}$ \\ ${ }^{*}$ David R. Cheriton School of Computer Science, University of Waterloo, Canada, Email: mlali@uwaterloo.ca \\ ${ }^{\dagger}$ Department of Electrical and Computer Engineering, University of Waterloo, Canada \\ Email: b7wu@uwaterloo.ca, pihan@bbcr.uwaterloo.ca \\ ${ }^{\ddagger}$ Dept. of Telecommunications and Media Informatics, Budapest University of Technology and Economics, Hungary \\ ${ }^{\S}$ Division of Mathematical and Computer Science, King Abdullah University of Science and Technology, Saudi Arabia
}

\begin{abstract}
Achieving instantaneous and precise failure localization in all-optical wavelength division multiplexing (WDM) networks has been an attractive feature of network fault management systems, and is particularly important when failuredependent protection is employed. The paper introduces a novel framework of real-time failure localization in all-optical WDM mesh networks, called monitoring-burst (m-burst), which aims to initiate a graceful compromise between consumed monitoring resources and monitoring delay. Different from any previously reported solution, the proposed $m$-burst framework has a single monitoring node (MN) which launches optical bursts along a set of pre-defined close-loop routes, called monitoring cycles (m-cycles), to probe the fiber links along the m-cycles. Bursts along different $\mathbf{m}$-cycles are non-overlapping through any link of the network. By identifying the lost bursts due to single link failure events only, the MN can unambiguously localize the failed fiber link in atleast 3-connected networks. We will justify the feasibility and applicability of the proposed m-burst framework in the scenario of interest. In order to avoid possible collision among optical bursts launched by the $\mathrm{MN}$, we define the problem of collision-free scheduling and formulate it into an integer linear program (ILP) in order to minimize the monitoring delay. Numerical results demonstrate the effectiveness of the proposed framework and the proposed solution.
\end{abstract}

\section{INTRODUCTION}

A network operator is committed up to $99.999 \%$ (five 9s) availability of connections as a part of service level agreements with its customers. High availability of network backbone is ensured by using fault detection, fault localization and traffic protection/restoration techniques. Instantaneous and precise failure localization in all-optical WDM mesh networks is essential in enabling fast and automatic failure-dependent restoration in the event of the failure of any shared risk link group (SRLG), which in turn serves as a key functional block in achieving an efficient and effective fault management system.

In the absence of the electronic data processing at the intermediate nodes, one of the most common methods to achieve instantaneous failure localization in all-optical WDM networks is via out-of-band monitoring, where a set of supervisory lightpaths (S-LPs) are launched and closely monitored at the receivers [1] - [9]. A receiver broadcasts an alarm in the control plane with the highest priority as soon as any irregularity such as loss-of-light (LoL) is detected [10]. By collecting the flooded alarms, the network controller or any routing entity, provided with a set of properly designed S-LPs, should be able to unambiguously localize the failed SRLG.

Link-based monitoring for the purpose is widely used in the current commercial carrier backbones by having each fiber link monitored via a single-hop S-LP. Thus, the number of required active monitors is in the order of the number of links of the network.

To reduce the number of monitors and to achieve solutions in the order of logarithm to the number of SRLGs, simple/nonsimple m-cycles, m-paths, and m-trails are investigated in [1] - [5]. These studies take the S-LP allocation problem as a topology coding process, where each SRLG is assigned a unique code. The $j$-th bit of the code of an SRLG is assigned 1 if the $j$-th S-LPs traverses through the SRLG, otherwise the bit is assigned 0 and the S-LP should be disjoint with the SRLG. When an SRLG fails, all the S-LPs passing through the SRLG are disrupted, which drive the associated monitors issuing alarms. Note that the alarms have to be flooded in the control domain since the monitors may be located at far away from the network controller or the routing entities. The network controller or the routing entities can possibly localize the failure only by collecting the flooded alarms to form a valid alarm code.

Obviously, the alarm dissemination and collection lead to additional management overhead and longer monitoring delay. To overcome the problem, the authors in [9] defined and investigated the scenario in which every $\mathrm{MN}$ has to individually perform unambiguous failure localization (UFL) based on locally available on-off status of the traversing SLPs via optical signal tapping. This is also referred to as local unambiguous failure localization (L-UFL) at each MN in which no alarm dissemination is required. Therefore under the scenario, the number of S-LPs, which stands for the length of alarm code and signaling dissemination overhead, is no longer of interest, while the amount of monitoring resources serves as the only performance metric. However, since each S-LP has to traverse through an $\mathrm{MN}$ such that the $\mathrm{MN}$ can be L-UFL capable, the amount of monitoring resources (in terms of required wavelength channels for the S-LPs) could be significantly increased due to the additional routing constraint for the S-LPs. This problem becomes more serious when failures of multi-link SRLGs are considered. According to our 
early assessment [8], a feasible UFL solution takes 10 or more wavelength channels on average along each link when all the SRLGs with up to 3 links are considered.

To reduce monitoring resource consumption, time division multiplexing (TDM) of S-LPs at intermediate nodes could be used. But that would require expansive $\mathrm{O} / \mathrm{E} / \mathrm{O}$ conversion of S-LPs at the intermediate nodes. Moreover, it would go against transperency of the all-optical network.

This paper aims to solve the abovementioned problem of high monitoring resource requirement by introducing a novel framework of all-optical failure localization, called monitoring burst (m-burst). With the m-burst framework, a single $\mathrm{MN}$ is allocated with a set of close-loop S-LPs (or termed m-cycles in the following context). The MN launches optical bursts along the m-cycles to detect the on-off status of the m-cycles. Due to the close-loop shape of an m-cycle, the launched optical burst will be received by the $\mathrm{MN}$ in case all the fiber links along the $\mathrm{m}$-cycle are working properly, and the burst will be lost if any fiber link along the m-cycle has failed. Thus different from the scenario of L-UFL defined in [9] where an MN relies on the on-off status of each traversing S-LP to determine each bit in the alarm code, the MN of the m-burst framework determines each bit of the alarm code based on whether the corresponding burst is received at the expected time instant or not.

Since each m-burst is a short-duration optical flow, we no longer need to statically reserve a whole wavelength channel along each link of an m-cycle; on the other hand, multiple optical bursts can be multiplexed in the time domain on the same wavelength channel, which is expected to significantly reduce the consumed monitoring resources. Such saving of monitoring resources, nonetheless, cannot be achieved without additional complications and overhead. Due to lack of optical buffers, an optical burst has a deterministic arrival and departure time instants at each intermediate node once it is launched at the MN. Thus, it is possible that two m-bursts collide at any link traversed by both the corresponding m-cycles. Note that the proposed m-burst framework has an essential prerequisite that any burst loss should be due to fiber link cut instead of any other reason such as burst collision, otherwise the MN would be subject to a false alarm or an illegal alarm code. Provided with $k$ wavelengths (full wavelength convertibility assumed) along each link for supporting the failure localization system, burst collision can be avoided only if no more than $k$ bursts have their traversal time periods overlapped with the others' along any link. Therefore, an immediate problem stemmed from the proposed m-burst framework is how to completely avoid any burst collision.

In this paper, we set our goal as to claim the feasibility of the proposed m-burst framework by formulating the burst collision avoidance task as a burst scheduling problem. In order to completely avoid any possible burst collision, our strategy is to manipulate the timing of launching each optical burst at the MN. The paper focuses on the scenario of singlelink SRLGs with a single MN, where each link requires at most a single supervisory wavelength channel in each direction for the failure localization system.
The rest of the paper is organized as follows. Section II further details the proposed m-burst framework and the related implementation issues. In Section III, an integer linear program (ILP) is formulated to obtain burst collision-free scheduling under the proposed framework. A simple experiment on a small network is conducted, and the result is given in Section IV. Section V concludes the paper.

\section{M-BURST FrAMEWORK}

\section{A. Overviews on S-LP Allocation Problem}

The S-LP allocation problem is to find a trail expressed as a sequence of edges (links) $\left(v_{0}, v_{1}\right),\left(v_{1}, v_{2}\right), \ldots,\left(v_{n-2}, v_{n-1}\right),\left(v_{n-1}, v_{n}\right)$ in the network $G=(V, E)$, where $\left(v_{i}, v_{i+1}\right) \neq\left(v_{j}, v_{j+1}\right)$ if $i \neq j$, and $\left(v_{i}, v_{i+1}\right) \in E$ for $i=0, \ldots, n-1$. By properly designing a set of S-LPs with respect to the SRLGs under consideration, the on-off status of the S-LPs can form a valid alarm code mapped to a failure event on any one of the SRLGs.

An S-LP is a non-simple lightpath with a pair of transmitter and receiver (denoted as $T$ and $R$, respectively) along with a monitor at the receiver. As shown in Fig. 1(a), the S-LP can be pre-cross-connected in either $T \rightarrow a \rightarrow b \rightarrow c \rightarrow a \rightarrow$ $d \rightarrow e \rightarrow R$ or $T \rightarrow a \rightarrow c \rightarrow b \rightarrow a \rightarrow d \rightarrow e \rightarrow R$.

A supervisory optical signal is launched in the $\mathrm{m}$-trail. If any link traversed by an S-LP fails, the optical signal is disrupted, where the monitor will generate an alarm and broadcast the alarm in the optical network control domain.

Generally, a solution consists of a set of $J$ S-LPs $t_{1}, t_{2}, \ldots, t_{J}$, each corresponding to a specific bit position in the alarm code, denoted as $\left[a_{1}, a_{2}, \ldots, a_{J}\right]$, where $a_{j}=1$ means that the monitor on $t_{j}$ alarms and $a_{j}=0$ otherwise. Fig. 1(b) shows an example with three S-LPs $t_{1}, t_{2}, t_{3}$. If link $(0,1)$ fails, the monitors on $t_{1}$ and $t_{3}$ will alarm to produce the alarm code $[1,0,1]$. Similarly, if link $(0,2)$ fails, the monitors on all the three $\mathrm{m}$-trails will alarm and the resulting alarm code is $[1,1,1]$. The alarm code table (ACT) in Fig. 1(c) maintains the mapping between an alarm code and any failure event.

It is possible to have any constraint in the S-LP allocation process to satisfy specific design requirement. Under the mburst framework, we require each S-LP, called m-cycle, to be a close-loop cycle and traverse through a designated MN node. We will demonstrate our solution to the constrained SLP allocation problem via the ILP formulation in Section III.

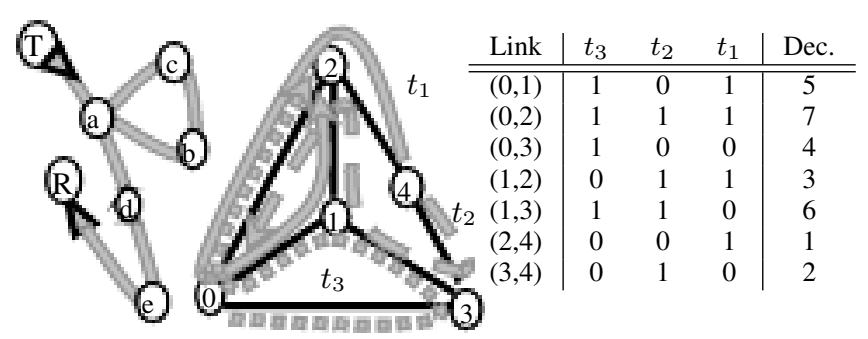
(a) m-trail
(b) An m-trail solution
(c) Alarm code table 


\section{B. Framework Descriptions}

With the proposed m-burst framework, the $\mathrm{MN}$ is given and a set of m-cycles are identified, where an optical burst with a fixed length is launched along each m-cycle in order to determine if there is any failed link along the m-cycle. With the short-duration bursts, the monitoring resources can be reduced by multiplexing the burst traversals of multiple $\mathrm{m}$-cycles in the time domain along some wavelength links. Therefore, the proposed m-burst is expected to significantly improve the performance reported by the previous studies where static S-LPs are employed.

Although with such saving of monitoring resources, m-burst is subject to longer monitoring delay and larger signaling overhead. The longer monitoring delay is due to the discrete time instants on burst return to the $\mathrm{MN}$ and any possible mechanism for ensuring that a burst loss is merely due to link failure instead of any other reason. The larger signalling overhead, on the other hand, is caused by the signalling effort for real-time configuration of switch fabrics for the m-cycles. Therefore, a careful design is required to mitigate the above two issues.

Here, the monitoring delay is defined as the shortest duration starting when the MN launches the first burst until it can come up with a valid alarm code (including the code 00000 showing no failure). With m-bursts, monitoring delay is an index for how fast the MN can respond to any failure event considered in the m-cycle deployment, while the number of m-cycles and their total cover length concern the amount of signaling overhead in switching fabric configuration.

Different from the case with static S-LPs, the proposed mburst framework should be supported by a specific resource reservation scheme. We suggest to using the Just-EnoughTime (JET) scheme [11] that takes advantage of tell-andgo signalling and delayed reservation scheme. With delayed reservation, the source node sends the control packet first in order to reserve switching fabric at each intermediate node of the route, and the optical burst is launched after an offset time. With tell-and-go, the offset time is minimized by sending the optical burst without waiting for the acknowledgement; thus the offset time can be as short as simply the sum of nodal process time at each intermediate node. Thus, JET has each node specified with the exact time instants that the optical burst will arrive and depart, respectively, such that the switching fabric of the node will be reserved only when the optical burst traverses through the node, and will be released after the departure of the burst without any further signalling notice.

In the m-burst framework, $\mathrm{MN}$ can be considered as only edge node and all other nodes of the network as core nodes. Most of the activities of traditional OBS are done as preprocessing in the framework. Burst assembling become trivial because bursts are fixed length optical signals. One monitoring wavelength channel at each direction of each link is reserved. The routes of the m-cycles are found as the ILP solution. Contention for resources across the network is resolved by scheduling m-burst launching times from MN. As the routes of the bursts are known and stable, calculation of burst offset from respective control packet also become trivial. Launching time of a control packet is calculated by deducting the corresponding offset from the burst launching time.

The remaining main functions for $\mathrm{MN}$ are sending control packets and bursts which can be considered as routine works. The only remaining work for the core nodes is resource scheduling. The estimated set-up and estimated release scheme for resource reservation can be used because burst size is fixed and control packets carry offset information.

Although we use same resource reservation mechanism as JET, a non-trivial difference exists between the two in the aspect of design premises and implementations. The bursts in the proposed m-burst framework are taken as a mean of out-of-band monitoring that inspect the on-off status along the m-cycles. Instead of bearing any significant modulated information bit for data transfer as in OBS, m-bursts are fixed length optical signals carrying minimal information such as identification of m-cycles. With this regards, a much relaxed requirement on time precision and optical signal quality can be expected, which makes the proposed framework easily implementable.

\section{Problem Definition}

An immediate problem raised under the m-burst framework is the timing for launching the burst for each m-cycle, such that there is not any collision (or burst contention in the context of OBS) in the process of resource reservation. A burst collision simply yields an incorrect monitoring and failure location result. Note that the $\mathrm{MN}$ is the only sender and receiver of the bursts for all the m-cycles. Therefore, a well designed scheduling scheme for the time instant of launching the bursts at the MN is expected to nicely resolve any collision at any link. Without the scheduling effort, collision may occur among the bursts of any $k+1 \mathrm{~m}$-cycles that traverse through a common link having $k$ reserved wavelengths.

There are two extreme cases in the scheduling problem. One is that each link contains sufficient wavelength channels for monitoring, such that the MN can launch all the optical bursts at the same time without any collision. In this case, the monitoring delay is simply the delay along the longest $\mathrm{m}$ cycle. The other extreme is that the MN conservatively keeps no more than one burst on-the-fly in the network, where an optical burst is launched after confirming the probing result of the previously launched burst. This obviously can avoid any collision under any circumstance while at the expense of taking the longest possible monitoring delay which is the sum of the delays of all the m-bursts.

In this paper, we investigate the scenario of single link failure localization where every link has at most a single dedicated wavelength channel in each direction for the proposed failure localization framework, determine a set of m-cycles traversing through a given $\mathrm{MN}$ according to the network topology, and schedule optical burst launching from the MN along the $\mathrm{m}$ cycles avoiding any collision. 


\section{PROBLEM FORMULATION}

The section provides an ILP formulation for the joint design of $\mathrm{m}$-cycles and $\mathrm{m}$-burst scheduling provided with a single MN and at most a single monitoring wavelength channel in each direction of network links. The notation list is given below.

- $G$ : the network, $G=(V, E)$.

- $V$ : The set of all nodes in the network, $t, u$ and $v$.

- $E$ : The set of links in the network, $(t, u)$ and $(u, v)$.

- $M N$ : The monitoring node in the network, $M N \in V$.

- $r_{1}, r_{2}$ : Predefined cost ratios.

- $J$ : Predefined maximum number of allowed m-cycles in an ILP solution.

- $j, k$ : Indices of m-cycles $j, k \in\{0,1,2, \ldots, J-1\}$.

- $m^{j}$ : Binary variable, it is equal to 1 if the $j$-th m-cycle is in an ILP solution and 0 otherwise.

- $d_{u}^{j}$ : Binary variable, it is equal to 1 if node $u$ is the sink of $j$-th m-cycle and 0 otherwise. Please note that only $\mathrm{MN}$ can be sink of an m-cycle.

- $e_{u v}^{j}$ : Binary variable, it is equal to 1 if the $j$-th m-cycle traverses link $(u, v)$ and 0 otherwise.

- $\delta$ : Predefined small positive constant.

- $q_{u v}^{j}$ : Fractional variable, it is defined as voltage of the vector $u \rightarrow v$ for the link $(u, v)$. It assumes an arbitrary positive value if the $j$-th $\mathrm{m}$-cycle traverses link $(u, v)$ and 0 otherwise.

- $z_{u}^{j}$ : Binary variable, it is equal to 1 if the $j$-th m-cycle visits node $u$ and 0 otherwise. The variable with $\delta$ and $q$ helps to keep each m-cycle in an ILP solution a single connected cycle.

- $\alpha_{u v}$ : Integer variable, the unique decimal alarm code of the fiber link between nodes $u$ and $v$. Links $(u, v)$ and $(v, u)$ have the same decimal alarm code.

- $\beta$ : Predefined small positive constant.

- $f_{u v}^{x y}$ : Binary variable, it is equal to 1 if $\alpha_{u v}>\alpha_{x y}$ and 0 if $\alpha_{u v}<\alpha_{x y}$. The variable with $\beta$ helps to find unique decimal alarm code for each fiber link of the network.

- $n$ : Integer variable, it is the maximum number of $\mathrm{m}$ cycles traversed through any link $(u, v)$.

- $N$ : A large predefined number representing infinity.

- $b$ : Predefined constant burst length in ms.

- $c_{u v}$ : Predefined burst propagation delay in $\mathrm{ms}$ of an $\mathrm{m}-$ burst through the link $(u, v)$.

- $s^{j}$ : Variable burst launching time from MN along $j$-th $\mathrm{m}$-cycle in ms.

- $T$ : Variable total monitoring delay in ms.

- $h_{M N v}^{j}$ : Binary variable, it is equal to 1 if $(M N, v)$ is the head link of $j$-th m-cycle and 0 otherwise.

- $r_{t M N}^{j}$ : Binary variable, it is equal to 1 if $(t, M N)$ is the tail link of $j$-th m-cycle and 0 otherwise.

- $w_{u v}^{t u}$ : Binary variable, it is equal to 1 if $j$-th m-cycle traverses link $(t, u)$ immediately before traversing link $(u, v)$ and 0 otherwise. This is link continuity variable. It helps to calculate propagation delays properly along non-simple m-cycles from MN to the network nodes.

- $p^{j}$ : A variable, it is total round trip burst propagation delay in ms along $j$-th m-cycle.

- $p_{u v}^{j}$ : A variable, it is burst propagation delay in ms along the $j$-th m-cycle from MN to on-cycle node $u$ when outgoing link from $u$ is on-cycle link $(u, v)$ and $N$ otherwise.

- $g_{u v}^{j k}$ : Binary variable, it is equal to 1 if $j$-th burst arrives to node $u$ before $k$-th burst i.e., $\left(s^{j}+p_{u v}^{j}\right)<\left(s^{k}+p_{u v}^{k}\right)$ and 0 if $j$-th burst arrives to the node after $k$-th burst i.e., $\left(s^{j}+p_{u v}^{j}\right)>\left(s^{k}+p_{u v}^{k}\right)$. It helps collision-free burst propagation through network links.

The specific ILP formulation is provided as follows. Objective:

$$
\text { Minimize }\left\{T+r_{1} * \sum_{j} m^{j}+r_{2} * n\right\}
$$

Subject to the following constraints:

A single sink is allowed for an m-cycle

$$
\sum_{u \in V} d_{u}^{j} \leq 1, \quad \forall j
$$

Only MN can be the sink of an m-cycle

$$
d_{M N}^{j}=\sum_{u \in V} d_{u}^{j}, \quad \forall j
$$

A sink can be assigned only to an m-cycle in an ILP solution

$$
m^{j} \geq d_{M N}^{j}, \quad \forall j
$$

Indices of the m-cycles in an ILP solution are lower than indices of the m-cycles not in the solution

$$
m^{j} \geq m^{j+1}, \quad \forall j: j \leq J-2
$$

A lower bound on the number of m-cycles in a solution is given to help the ILP solver to find the optimal solution faster

$$
\sum_{j} m^{j} \geq\left\lfloor\log _{2}|E|\right\rfloor+1
$$

An m-cycle can traverse a fiber link between 2 nodes in one direction only

$$
e_{u v}^{j}+e_{v u}^{j} \leq 1, \quad \forall(u, v) \in E, \forall j
$$

This is the flow conservation defined for each node of the network. It enforces that each monitoring structure consists of cycle(s)

$$
\sum_{(u, v) \in E}\left(e_{u v}^{j}-e_{v u}^{j}\right)=0, \quad \forall u \in V, \forall j
$$

A node is visited by an m-cycle if any adjacent link of the node is traversed by the m-cycle

$$
z_{u}^{j} \geq e_{u v}^{j}+e_{v u}^{j}, \quad \forall u \in V:(u, v) \in E, \forall j
$$


Voltages of the vectors corresponding to on-cycle links can be assigned non-zero values

$$
q_{u v}^{j} \leq e_{u v}^{j}, q_{v u}^{j} \leq e_{v u}^{j}, \quad \forall(u, v) \in E, \forall j
$$

This is the voltage constraint to make an m-cycle in an ILP solution a single connected cycle by ensuring that if an m-cycle traverses a node except the sink, the sum of its outgoing voltage values will be greater than the sum of its incoming voltage values. The specific voltage values are not important

$$
d_{u}^{j}+\sum_{(u, v) \in E}\left(q_{u v}^{j}-q_{v u}^{j}\right) \geq \delta * z_{u}^{j}, \quad \forall u \in V, \forall j
$$

All decimal link alarm codes must be positive integers

$$
\alpha_{u v} \geq 1, \quad \forall(u, v) \in E
$$

Decimal alarm code for a fiber link is based on the m-cycles traversing the link in either direction

$$
\alpha_{u v}=\sum_{j} 2^{j} *\left(e_{u v}^{j}+e_{v u}^{j}\right), \quad \forall(u, v) \in E
$$

Constraints (14) and (15) ensure a unique decimal alarm code for each link

$$
\begin{gathered}
\beta+\beta *\left(\alpha_{u v}-\alpha_{x y}\right) \leq f_{u v}^{x y}, \\
\forall(u, v),(x, y) \in E:(u, v) \neq(x, y) \\
\beta+\beta *\left(\alpha_{x y}-\alpha_{u v}\right) \leq 1-f_{u v}^{x y}, \\
\forall(u, v),(x, y) \in E:(u, v) \neq(x, y)
\end{gathered}
$$

Calculates the maximum number of m-cycles traversing through link $(u, v)$

$$
n \geq \sum_{j} e_{u v}^{j}, \quad \forall(u, v) \in E
$$

The lower and upper bounds of burst launching time. Burst starting times of only m-cycles in an ILP solution can be assigned positive values

$$
0 \leq s^{j} \leq m^{j} * N, \quad \forall j
$$

Calculation of total round trip burst propagation delay through $j$-th m-cycle

$$
p^{j}=\sum_{(u, v) \in E} c_{u v} * e_{u v}^{j}, \quad \forall j
$$

Finding collision free monitoring delay which is equal to the largest elapse time for any m-burst from the start of the monitoring period and return of the last burst back to the MN

$$
T \geq s^{j}+p^{j}+b, \quad \forall j
$$

An on-cycle link from the MN can be head link of the $j$-th m-cycle

$$
h_{M N v}^{j} \leq e_{M N v}^{j}, \quad \forall j
$$

Only an m-cycle in an ILP solution has a head link from the MN

$$
\sum_{(M N, v) \in E} h_{M N v}^{j}=m^{j}, \quad \forall j
$$

An on-cycle link to the MN can be tail link of the $j$-th m-cycle

$$
r_{t M N}^{j} \leq e_{t M N}^{j}, \quad \forall j
$$

Only an m-cycle in an ILP solution has a tail link to the MN

$$
\sum_{(t, M N) \in E} r_{t M N}^{j}=m^{j}, \quad \forall j
$$

Link continuity variable through node $u$ can be assigned 1, if the incoming link to the node is an on-cycle link of $j$-th m-cycle

$$
w_{t u v}^{j} \leq e_{t u}^{j}, \quad \forall u \in V:(t, u) \in E, \forall j
$$

Link continuity variable for the incoming tail link to $\mathrm{MN}$ and outgoing head link from MN of $j$-th m-cycle is ensured to be 1

$$
w_{t M N v}^{j}+\left(1-r_{t M N}^{j}\right) \geq h_{M N v}^{j}, \forall(M N, v) \in E, \forall j
$$

Only one link continuity variable is allowed to be 1 for an on-cycle incoming link for $j$-th m-cycle to node $u$

$$
\sum_{(u, v) \in E} w_{t u v}^{j}=e_{t u}^{j}, \quad \forall u \in V:(t, u) \in E, \forall j
$$

Only one link continuity variable is allowed to be 1 for an on-cycle outgoing link for $j$-th $\mathrm{m}$-cycle from node $u$

$$
\sum_{(t, u) \in E} w_{t u v}^{j}=e_{u v}^{j}, \quad \forall u \in V:(u, v) \in E, \forall j
$$

Constraints (24), (25), (26) and (27) identify a unique on-cycle incoming link to a node for each on-cycle out-going link from the node and allow calculation of propagation delays properly in presence of non-simple m-cycles in an ILP solution.

The lower and upper bounds of the burst propagation delay through the $j$-th m-cycle

$$
0 \leq p_{u v}^{j} \leq N, \quad \forall(u, v) \in E, \forall j
$$

The burst propagation delay toward the first link of $j$-th $\mathrm{m}$-cycle from $\mathrm{MN}$ is assigned as 0 . The constraint acts as a base case for the recursive burst propagation delay calculations as per constraints (30) - (33)

$$
p_{M N v}^{j} \leq\left(1-h_{M N v}^{j}\right) * N, \quad \forall(M N, v) \in E, \forall j
$$


The burst propagation delay along a link from a node $u$ is infinity if the link is not traversed by $j$-th m-cycle

$$
p_{u v}^{j} \geq\left(1-e_{u v}^{j}\right) * N, \quad \forall(u, v) \in E, \forall j
$$

When the node $u$ is not $\mathrm{MN}$, the constraint is for calculation of the propagation delay along $j$-th $\mathrm{m}$-cycle from the $\mathrm{MN}$ through the head link of the m-cycle to the node when the outgoing link of the m-cycle from the node is $(u, v)$

$$
\begin{aligned}
& p_{u v}^{j} \geq p_{t u}^{j}+c_{t u}-\left(1-w_{t u v}^{j}\right) * N, \\
& \forall(u, v),(t, u) \in E: u \neq M N, \forall j
\end{aligned}
$$

When the node $u$ is MN, the constraint is for the calculation of propagation delays along the $j$-th $\mathrm{m}$-cycle from the MN to itself when the outgoing link is $(M N, v)$. The burst propagation delay increases by the propagation delay of the incoming link if the outgoing link is not the head link of the m-cycle

$$
\begin{aligned}
& h_{M N v}^{j} * N+p_{M N v}^{j} \geq \\
& p_{t M N}^{j}+c_{t M N}-\left(1-w_{t M N v}^{j}\right) * N, \\
& \forall(M N, v),(t, M N) \in E, \forall j
\end{aligned}
$$

The total burst propagation delay of $j$-th m-cycle is the propagation delay up to the $\mathrm{MN}$ through the tail link of the m-cycle

$$
\begin{array}{r}
p^{j} \geq p_{t M N}^{j}+c_{t M N}-\left(1-r_{t M N}^{j}\right) * N, \\
\forall(t, M N) \in E, \forall j
\end{array}
$$

Constraint (34) and (35) enforce difference between arrival times of any two m-bursts along $j$-th and $k$-th m-cycles to node $u$ that are traversing same outgoing link $(u, v)$ from the node to the burst length $b$. Thus, they ensure collision free burst propagation through any link of the network. They are the key constraints for collision free burst scheduling.

$$
\begin{gathered}
\left(s^{j}+p_{u v}^{j}\right)-\left(s^{k}+p_{u v}^{k}\right) \geq\left(1-g_{u v}^{j k} * N\right) * b, \\
\forall u \in V:(u, v) \in E, \forall j, k: j \neq k \\
\left(s^{k}+p_{u v}^{k}\right)-\left(s^{j}+p_{u v}^{j}\right) \geq \\
\left(2 * g_{u v}^{j k}-1-\left(1-g_{u v}^{j k}\right) * N\right) * b, \\
\forall u \in V:(u, v) \in E, \forall j, k: j \neq k
\end{gathered}
$$

Objective (1) aims to minimize $T$, the monitoring delay. It also minimizes the number of $\mathrm{m}$-cycles and the maximum number of m-cycles traversing through any link in a single direction. Relative importance of the objectives is manipulated with the help of cost ratios $r_{1}$ and $r_{2}$.

Please refer to [4] for a thorough explanation of the constraints (2) - (15) which are related with finding m-cycles in an ILP solution. Constraint (16) is for finding the maximum number of m-cycles traverse through any link in a single direction. Constraints (17) - (35) are related with m-bursts scheduling.

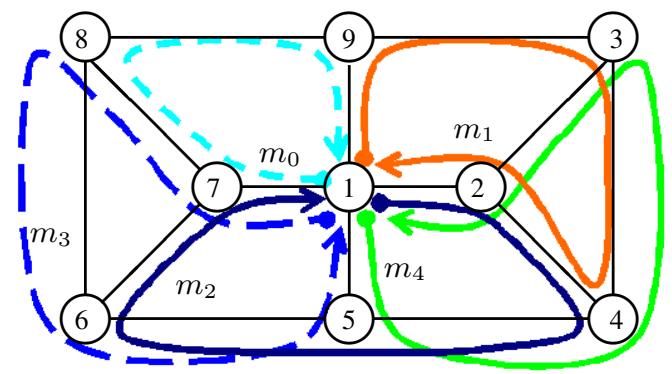

Fig. 2. M-cycle solution set for a network with 9 nodes and 14 links.

\section{NumericAl RESUlts}

We solved the ILP in modeling language GNU MathProg and translated the MathProg model using GLPK solver glpsol into a MPS file to run the program in ILOG CPLEX 11.1.

We assume that the burst length is $20 \mathrm{~ms}$, propagation delay in each link is $2 \mathrm{~ms}$, and there is only one wavelength channel along each direction of a link in a numerical experiment. We conduct the experiment on a network having 9 nodes and 14 links as shown in the Fig. 2. Node 1 is assigned as the MN.

The ILP solution has $5 \mathrm{~m}$-cycles for any single link failure localization. M-cycles $m_{0}, m_{1}, m_{2}, m_{3}$, and $m_{4}$ traverse 4, $5,6,5$, and 5 links, respectively. The solution allows at most $2 \mathrm{~m}$-cycles in a direction of a link of the network. A set of unique decimal alarm codes for the links $(1,2),(1,5),(1,7)$, $(1,9),(2,3),(2,4),(3,4),(3,9),(4,5),(5,6),(6,7),(6,8)$, $(7,8)$ and $(8,9)$ are given as $22,24,13,3,16,6,18,2,20$, $12,4,8,9$, and 1 , respectively.

The total propagation delays through m-cycles $m_{0}, m_{1}, m_{2}$, $m_{3}$, and $m_{4}$ are $8 \mathrm{~ms}, 10 \mathrm{~ms}, 12 \mathrm{~ms}, 10 \mathrm{~ms}$, and $10 \mathrm{~ms}$, respectively. The starting time of the bursts for the m-cycles $m_{0}, m_{1}, m_{2}, m_{3}$, and $m_{4}$ are $0 \mathrm{~ms}, 20 \mathrm{~ms}, 0 \mathrm{~ms}, 20 \mathrm{~ms}$, and $0 \mathrm{~ms}$, respectively. Thus $T$ is derived either from m-cycle $m_{1}$ as $s^{1}+p^{1}+b=20+10+20=50 \mathrm{~ms}$ or from m-cycle $m_{3}$ as $s^{3}+p^{3}+b=20+10+20=50 \mathrm{~ms}$.

Figure 3 shows m-burst traversal timing through each link traversed by $2 \mathrm{~m}$-bursts in the same direction. A rectangle represents the time period that the corresponding m-burst traverses through a link. The time period is equal to $p_{u v}^{j}+b$ $=2+20=22 \mathrm{~ms}$. All 3 links are traversed by $2 \mathrm{~m}$-bursts back to back which means 2 bursts traverse through a link one immediately after the other. Back to back periods are indicated by black rectangles. As shown in the figure, bursts for the mcycles $m_{0}$ and $m_{3}$ traverse links $(1,7)$ and $(7,8)$ back to back, and bursts for the m-cycles $m_{4}$ and $m_{1}$ traverse link (2, 1) back to back.

With the scheduling effort, burst collision was completely avoided by manipulating launching time of each $\mathrm{m}$-burst at the $\mathrm{MN}$, and the resultant monitoring delay is $T=50 \mathrm{~ms}$, which can well incorporate with any fast restoration scheme with a failure-dependent protection plan. 


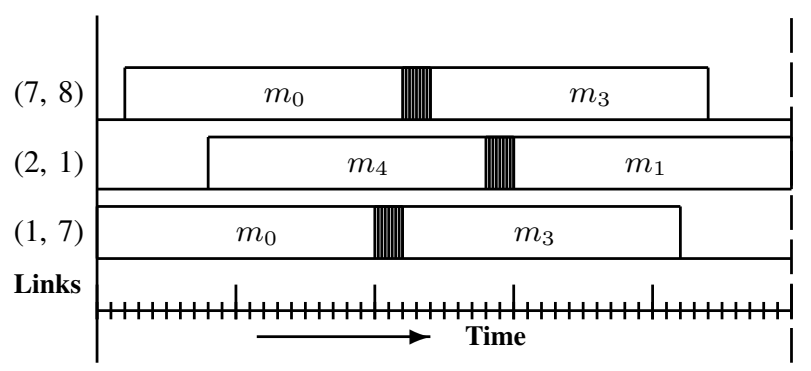

Fig. 3. M-burst scheduling for the network

\section{Conclusions}

The paper presented a novel framework of failure localization via out-of-band monitoring in all-optical WDM networks, called monitoring burst (m-burst), which aims to significantly reduce the consumed monitoring resources that are necessary in the previously reported studies. With the proposed mburst framework, the monitoring node $(\mathrm{MN})$ launches shortduration optical bursts along a set of cycles (or termed mcycles) to probe their on-off status. The MN thus can achieve unambiguous failure localization (UFL) for the single-link SRLGs failures.

The paper firstly defined the m-burst framework and identified the burst scheduling problem in order to completely avoid burst collision. Then, an ILP was formulated for a joint design of the m-cycles and derivation of the timing of launching the bursts along each m-cycle, aiming to achieve the minimum monitoring delay and signaling overhead. As a preliminary study on the proposed framework, we conducted a simple numerical experiment on a small network, and the result demonstrated feasibility and easy implementation of the framework.

With the m-burst framework, our future work is identified as follows: (1) investigate the effect of burst length on monitoring delay; (2) devise a heuristic algorithm to find near optimal solution of the problem; (3) investigate the scenario where more than one $\mathrm{MN}$ are present in the network and the MNs are either loosely synchronized or completely non-synchronized; and (4) investigate the tradeoff between delay and monitoring resources.

\section{REFERENCES}

[1] H. Zeng, C. Huang, A. Vukovic, and M. Savoie, "Fault Detection and Path Performance Monitoring in Meshed All-Optical Networks," IEEE GLOBECOM '04.

[2] B. Wu, and K. L. Yeung, "M2-CYCLE: an optical layer algorithm for fast link failure detection in all-optical mesh networks," in Proc. IEEE GLOBECOM '06, Dec. 2006, pp. 1-5.

[3] B. Wu, and K. L. Yeung, and P.-H. Ho, "Monitoring cycle design for fast link failure localization in all-optical networks," IEEE/OSA Journal of Ligthwave Technology, vol. 27, no. 10, pp. 1392-1401, May 2009.

[4] B. Wu, P.-H. Ho, and K. L. Yeung, "Monitoring Trail: on fast Link failure localization in all-optical WDM Mesh Networks," IEEE/OSA Journal of Ligthwave Technology, vol. 27, no. 18, pp. 4175-4185, Sept. 2009.

[5] J. Tapolcai, B. Wu, and P.-H. Ho, "On Monitoring and Failure Localization in Mesh All-Optical Networks," in Proc. IEEE InfoCom 09, Apr. 2009, pp. 1008-1016.
[6] S. Ahuja, S. Ramasubramanian, and M. Krunz, "Single link failure detection in all-optical networks using monitoring cycles and paths," IEEE/ACM Transactions on Networking (TON), vol. 17, no. 4, pp. 10801093, August 2009.

[7] H. Zeng, C. Huang, and A. Vukovic, "A novel fault detection and localization scheme for mesh all-optical networks based on monitoringcycles," Photonic Network Communications, vol. 11, no. 3, pp. 277-286, May 2006.

[8] J. Tapolcai, P. -H. Ho, and B. Wu, "Failure Localization for Shared Risk Link Groups in All-Optical Mesh Networks using Monitoring Trails", technical report available at http://bbcr.uwaterloo.ca/ pinhan/mtrail_SRLG.pdf.

[9] B. Wu, P. -H. Ho, J. Tapolcai, and X. Jiang, A Novel Framework of Fast and Unambiguous Link Failure Localization via Monitoring Trails, in IEEE Infocom 2010 WIP.

[10] S. Stanic, S. Subramaniam, H. Choi, G. Sahin, and H.-A. Choi, "On monitoring transparent optical networks." Int'l Conf. on Parallel Processing Workshops, August 2003, pp. 217-223.

[11] C. Qiao and M. Yoo, Optical burst switching (OBS) - a new paradigm for an optical Internet, Journal of High Speed Networks, vol. 8, no. 1, pp. 69-84, 1999. 